\title{
A Novel Simulation Model for Coded OFDM in Doppler Scenarios: DVB-T versus DAB
}

\author{
Mario Poggioni, Luca Rugini, Paolo Banelli \\ Dept. of Elect. and Inform. Eng. (D.I.E.I.) \\ University of Perugia \\ Perugia, Italy \\ \{mario.poggioni, luca.rugini, paolo.banelli\}@diei.unipg.it
}

\begin{abstract}
This paper develops a novel simulation model to characterize the bit error rate (BER) performance of coded orthogonal frequency-division modulation (OFDM) systems affected by Doppler spread. This simulation model, which acts directly in the frequency domain, greatly enhances the simulation efficiency, while maintaining a very good accuracy. By this model, the BER of two of the most popular wireless broadcasting standards, digital audio broadcasting (DAB) and terrestrial digital video broadcasting (DVB-T), has been assessed in Rayleigh and Rice channels. Simulation results show that the delay spread of the channel heavily affects the performance of DVB-T, while DAB is less sensitive to the frequency-selectivity of the channel. Moreover, DVB-T yields better performance than DAB in typical Rayleigh channels, whereas in Rice channels, for high signal-to-noise ratio (SNR), DAB outperforms DVB-T.
\end{abstract}

\section{Keywords-Coded OFDM; Doppler spread; DAB; DVB-T}

\section{INTRODUCTION}

Orthogonal frequency-division modulation (OFDM) is a well established and widely used technique for high-rate communications in frequency-selective fading channels, due to its easy per-subcarrier equalization in the frequency domain, and in particular it is used in many popular wireless standards, such as $802.16 \mathrm{e}, 802.11 \mathrm{a}$, terrestrial digital video broadcasting (DVB-T), and digital audio broadcasting (DAB) [1][2]. However, in high-mobility environments, the time variation (i.e., the Doppler spread) of mobile radio channels destroys the orthogonality of the OFDM subcarriers, leading to the so-called intercarrier interference (ICI) [3]. If advanced time-variant equalization techniques are not used, the ICI significantly degrades the performance of OFDM systems, which exhibit bit error rate (BER) floors that channel coding can only try to reduce. Consequently, a deep understanding of the ICI behaviour is of paramount importance in order to mitigate its effect as well as to assess analytically the BER performance.

Previous research on this topic [4]-[6] has shown that, for uncoded systems, the ICI can be well modelled as an additive white Gaussian noise (AWGN), and its average power was derived in closed form in [4][7]. However, a more appropriate figure of merit of a communication system is the coded BER, and consequently we are interested to model the ICI in coded systems. We will show that the AWGN model of the ICI in [6] is not adequate for assessing the BER performance of coded
OFDM systems. Specifically, it neglects the effects of the channel power delay profile, which actually does not affect the BER performance of uncoded systems [6][8], while it can greatly impact the coded BER performance. Indeed, we will show that the channel power delay profile has a major impact on the BER performance, even when the mobility is very low (and consequently the ICI could be neglected).

The broader scope of this work is to assess by simulations the behaviour of coded OFDM (COFDM) systems that adopt simple per-subcarrier equalization. This is obtained by comparing the Doppler resistance of the two most popular standards for mobile broadcasting, DAB and DVB-T. To the best of our knowledge, this is the first paper that presents a very deep comparison between DAB and DVB-T in mobile scenarios. This comparison is not straightforward because, even if these two standards are quite similar, they have distinctly different characteristics in respect of equalization (differential for DAB, coherent for DVB-T), channel coding (convolutional for $\mathrm{DAB}$, and concatenated Reed-Solomon (RS) with convolutional for DVB-T), and interleaving (long time interleaving for $\mathrm{DAB}$, much shorter interleaving for DVB-T).

A key point of this paper is the development of a simple and efficient simulation model which assesses with good accuracy the BER performance of both standards. In particular, we characterize the effects of the ICI, which should be necessarily taken into account in a fair comparison between DAB and DVB-T. This is obtained by introducing an equivalent frequency-domain OFDM model (EFDOM), which does not replicate all the OFDM TX-RX chain. A specific merit of our model is its capability to reduce the simulation time of a factor from 2 to 15 (depending on the channel model and on the code rate) with respect to the classical simulation of OFDM systems.

We will thus give an insight on the merits and flaws of the two broadcasting standards, pointing out which is the most adequate for transmissions in high-mobility scenarios. We will try to make the comparison as fair as possible, by choosing the same constellation order (quadrature phase shift keying (QPSK) for DVB-T, differential QPSK (DQPSK) for DAB) and analogous coding rate (CR). However, similar considerations can be extended to higher order constellations for DVB-T. We also considered imperfect channel estimation in the DVB-T receiver, for a "fair" comparison with DAB, which is implicitly equipped by a channel equalizer by means of a differential demodulator [2]. It is well known that the ICI 
effects are more evident as much the subcarriers get closer. Thus, we choose the two modes for DAB and DVB-T that are more resistant to Doppler effects and are characterized by the larger subcarrier spacing and thus the lower fast Fourier transform (FFT) size $N$.

The rest of this paper is organized as follows. Section II briefly describes the channel model, general OFDM systems in time-varying channels and both DVB-T and DAB physical layers. In Section III, we describe some channel estimation techniques for DVB-T. In Section IV, we develop our simple EFDOM for the system of interest, and in Section $V$ we compare DAB and DVB-T by means of simulation results.

Notation: Bold uppercase (lowercase) letters denote matrices (column vectors); the superscripts ${ }^{*},{ }^{T},{ }^{H}$ denote conjugation, transpose, Hermitian transpose, while $\|\cdot\|^{2}$ denote Frobenius norm. We use $[\mathbf{X}]_{i, j}$ to denote the $(i, j)$-th entry of the matrix $\mathbf{X} ;[\mathbf{X}]_{i}\left([\mathbf{x}]_{i}\right)$ denotes the $i$-th row (element) of the matrix $\mathbf{X}$ (vector $\mathbf{x}$ ), while $|\mathbf{x}|$ is the vector that contains the absolute values of the elements of $\mathbf{x} ; \mathbf{I}_{K}$ and $\mathbf{1}_{K}$ denote the identity matrix and the all-ones (column) vector of size $K$, respectively. $\operatorname{Diag}(\mathbf{a})$ is a diagonal matrix with the entries of $\mathbf{a}$ on the diagonal, whereas $\operatorname{diag}(\mathbf{A})$ is the column vector containing the main diagonal of $\mathbf{A}$; finally, $E[\cdot]$ denotes statistical expectation.

\section{SYSTEM MODEL}

\section{A. Channel Model}

We have modelled the channel according to the COST 207 [9] standard, as wide-sense stationary with uncorrelated scattering (WSSUS) [10]. For Rayleigh channels we have used the Bad Urban (BU) profile, while for Rice channels we have adopted the Rural Area (RA) profile. Although the RA is characterized by a Rice factor $K=0 \mathrm{~dB}$, we have also considered other values of $K$. We have generated the time variability of the channel taps with the sum-of-sinusoids method described in [11].

\section{B. OFDM System Model}

Consider a classical OFDM system with $N$ subcarriers and cyclic prefix (CP) of length $L_{C P} \geq L$, where $L$ is the number of channel taps. We denote by $h_{l}[k]$ the $l$-th discrete-time channel tap at time $k$, with power $\sigma_{l}^{2}$. The received vector $\mathbf{y}(n)$ in the frequency domain is expressed by

$$
\mathbf{y}(n)=\mathbf{F} \tilde{\mathbf{H}}(n) \mathbf{F}^{H} \mathbf{x}(n)+\mathbf{w}(n)=\mathbf{y}_{U}(n)+\mathbf{n}_{I C I}(n)+\mathbf{w}(n),
$$

where $n$ is the block index, $\mathbf{F}$ is the $N \times N$ unitary DFTmatrix, $\mathbf{x}(n)$ is the transmitted data vector, $\mathbf{w}(n)$ is the thermal AWGN and $\widetilde{\mathbf{H}}(n)$ is the channel convolution matrix associated with the time-varying channel. The terms

$$
\begin{aligned}
& \mathbf{y}_{U}(n)=\mathbf{H}_{U}(n) \mathbf{x}(n)=\mathbf{F} \tilde{\mathbf{H}}_{U}(n) \mathbf{F}^{H} \mathbf{x}(n) \\
& \mathbf{n}_{I C I}(n)=\mathbf{H}_{I}(n) \mathbf{x}(n)=\mathbf{F} \tilde{\mathbf{H}}_{I}(n) \mathbf{F}^{H} \mathbf{x}(n)
\end{aligned}
$$

represent the useful received signal and the ICI introduced by the time-variant part of the channel $\widetilde{\mathbf{H}}_{I}(n)$, respectively, and it holds true

$$
\begin{gathered}
\mathbf{H}_{U}(n)=\operatorname{Diag}\left(\sqrt{N} \mathbf{F} \overline{\mathbf{h}}_{T}(n)\right), \\
\text { where we define } \quad \overline{\mathbf{h}}_{T}(n)=\frac{1}{N} \sum_{i=n\left(N+L_{C P}\right)+L_{C P}+1}^{(n+1)\left(N+L_{C P}\right)} \mathbf{h}_{T}(i), \quad \text { and }
\end{gathered}
$$

$\mathbf{h}_{T}(i)=\left[h_{0}(i), \ldots, h_{L-1}(i)\right]^{T}$.

It is worth noting that $E\left[\left|\mathbf{y}_{U}(n)\right|^{2}\right]=\mathbf{1}_{N}-E\left[\left|\mathbf{n}_{I C I}(n)\right|^{2}\right]$ $=\left(1-P_{I C I}\right) \mathbf{1}_{N}$, with $^{1}[4]$

$$
P_{I C I}=1-\frac{1}{N^{2}}\left[N+2 \sum_{n=1}^{N-1} n J_{0}\left(2 \pi \bar{f}_{D} \frac{N-n}{N}\right)\right] .
$$

In (4), $\bar{f}_{D}$ represents the normalized Doppler rate, defined by $\bar{f}_{D}=f_{D} / \Delta f=N T_{S} f_{C} v / c$, where $f_{D}$ represents the maximum Doppler spread, $\Delta f$ is the subcarrier separation, $f_{C}$ is the carrier frequency, $c$ represents the light speed, and $v$ stands for the vehicle speed in the antenna direction. For simplicity, guard bands are not considered. The power in (4) is the mean power of the ICI on each subcarrier, unless for the few edge ones, whose effect is negligible.

In this work we focus on two transmission standards, DVB-T [1] and DAB [2]: for DVB-T we consider the $2 \mathrm{~K}$ subcarriers case (both for 5 and $8 \mathrm{MHz}$ bandwidths), while for DAB we consider mode III (with $N=256$ subcarriers). Table I summarizes the key parameters of the two systems we

\begin{tabular}{|c|c|c|}
\hline & DAB - mode III & DVB-T $5 \div 8 \mathrm{MHz}$ \\
\hline Bandwidth (MHz) & $\mathbf{1 . 5 ( 1 . 5 3 6 )}$ & $\mathbf{5}(4.71)-\mathbf{8}(7.61)$ \\
\hline Active subcarriers $N_{A}$ & 192 & 1705 \\
\hline Carrier spacing $\Delta f(\mathbf{K H z})$ & 8 & $2.79-4.46$ \\
\hline Data block duration $T_{u}(\mu \mathrm{s})$ & 125 & 358.40 - 224 \\
\hline CP duration $T_{c p}(\boldsymbol{\mu s})$ & $31.25\left(T_{u} / 4\right)$ & $\begin{array}{c}22.40-14\left(T_{u} / 16\right) \\
11.20-7\left(T_{u} / 32\right)\end{array}$ \\
\hline Sampling freq. $F_{S}(\mathrm{MHz})$ & 2.048 & $\begin{array}{l}\text { 40/7 (5.714) } \\
64 / 7(9.14)\end{array}$ \\
\hline Modulation & $\begin{array}{l}\pi / 4 \text {-shifted } \\
\text { DQPSK }\end{array}$ & QPSK \\
\hline Net bit-rate (Mbps) & $\begin{array}{l}1.152(\mathrm{CR}=1 / 2) \\
1.536(\mathrm{CR}=2 / 3)\end{array}$ & $\begin{array}{l}3.77-\mathbf{6 . 0 3}(\mathrm{CR}=1 / 2) \\
\mathbf{5 . 0 3}-\mathbf{8 . 0 4}(\mathrm{CR}=2 / 3)\end{array}$ \\
\hline $\begin{array}{c}\text { Spectral efficiency } \\
(\text { bit } / \mathbf{s} / \mathbf{H z})\end{array}$ & $\begin{array}{c}\mathbf{0 . 5 6 2}(\mathrm{CR}=1 / 2) \\
\mathbf{0 . 7 5}(\mathrm{CR}=2 / 3)\end{array}$ & $\begin{array}{c}\mathbf{0 . 6 6}-\mathbf{0 . 7 5}(\mathrm{CR}=1 / 2) \\
\mathbf{0 . 8 8}-\mathbf{1}(\mathrm{CR}=2 / 3)\end{array}$ \\
\hline
\end{tabular}
consider herein, where $T_{u}$ is the duration of the OFDM symbol without CP, $T_{c p}$ is the duration of the CP, $F_{S}$ is the sampling frequency.

TABLE I

SYSTEM PARAMETERS

\section{DVB-T CHANNEL ESTIMATION AND EQUALIZATION}

For the sake of complexity reduction, in this paper we consider only linear channel estimation techniques that exploits the observation of a single OFDM symbol. For the same reason, we do not exploit any advanced technique [8] [12] to compensate for the ICI. Consequently, we consider a per-subcarrier equalization where for each QPSK symbol received on a given subcarrier we compensate only for the estimated phase of the channel, because this way we take implicitly into account the channel state information (CSI) of the channel, thus allowing a better exploitation of soft convolutional decoding techniques [13]. We briefly summarize the channel interpolation techniques considered for DVB-T.

1 In the case of DVB-T, pilots have higher power than data; this can be accounted for, without significant loss of accuracy, with a suitable multiplicative correction factor 1.088 applied to $P_{I C I}$. 
Linear interpolation (LI): for each subcarrier, the frequencydomain channel response is obtained by a linear interpolation of the values estimated on the two closest pilot subcarriers.

Least-squares (LS) interpolation (LSI): this is the maximumlikelihood (ML) estimator [14] and the minimum variance unbiased (MVU) estimator [15] for the case of interest, i.e., without prior knowledge of the channel statistics and performing the estimate within a unique OFDM symbol. Assuming that the number of channel taps $L$ is known, then, if $\overline{\mathbf{x}}_{p}$ is the pilot vector, $\mathbf{F}_{L}$ the submatrix containing the first $L$ columns of $\mathbf{F}$, and $\mathbf{F}_{p}$ the submatrix containing the $p$ equispaced rows of $\mathbf{F}_{L}$ corresponding to the pilots positions in the given block, the LS estimator is expressed by

$$
\tilde{\mathbf{x}}_{L S}=\mathbf{F}_{L}\left(\mathbf{F}_{p}^{H} \mathbf{F}_{p}\right)^{-1} \mathbf{F}_{p}^{H} \overline{\mathbf{x}}_{p} .
$$

\section{SIMPlified SimUlation MODEL}

In order to statistically characterize the behaviour of the considered OFDM systems in time varying channels and to allow a simulation of the overall coded system with a reduced complexity (i.e., simulation time and memory requirements), we have derived a simple, accurate and sufficiently flexible frequency-domain equivalent model. To this end, as in [16][18], in this section we focus on the DVB-T inner system, i.e., the DVB-T system without RS coding, in order to simulate a low BER in a reasonable time for most of the scenarios of interest ${ }^{2}$. Our model is based on the following three assumptions:

A1) There is no performance loss in considering codewords with length equal to the interleaver depth, i.e., with a length that is lower than the actual DVB-T codeword length. This is motivated by the forced termination of the trellises usually employed in the Viterbi decoding to attain a manageable complexity [19];

A2) The average powers of the useful received signal and of the ICI can be assumed uncorrelated for DVB-T and DAB as expressed by (see eq. (2))

$$
E\left[\left\|\mathbf{y}_{U}(n)\right\|^{2}\left\|\mathbf{n}_{I C I}(n)\right\|^{2}\right]=E\left[\left\|\mathbf{y}_{U}(n)\right\|^{2}\right] E\left[\left\|\mathbf{n}_{I C I}(n)\right\|^{2}\right] \text {; }
$$

A3) The ICI effect can be summarized by the knowledge of its autocorrelation matrix and of the pdf of the average power of the ICI during an OFDM block.

With the assumptions A1-A3, we can simulate a simplified system statistically equivalent ${ }^{3}$ to the actual one, by generating only few parameters and completely in the frequency domain. Dropping hereafter the block index $n$ for notation simplicity, the general model can be written as

$$
\mathbf{y}^{(D V B-T)}=\mathbf{H}_{E F D O M}^{(D V B-T)} \overline{\mathbf{x}}+\mathbf{w}+\sqrt{\varphi} \tilde{\mathbf{n}}_{I C I},
$$

where $\mathbf{y}$ is the received signal vector, $\mathbf{H}_{E F D O M}^{(D V B-T)}$ is a suitable diagonal matrix, obtained by the useful channel matrix $\mathbf{H}_{U}$ in (1) by selecting the $N_{A}$ elements of $\operatorname{diag}\left(\mathbf{H}_{U}\right)$ that correspond to the active subcarriers (see Table I), $\overline{\mathbf{x}}$ is the QPSK data vector before symbol interleaving [1], $\mathbf{w}$ is a

\footnotetext{
${ }^{2}$ However, in some scenarios it is possible to simulate in reasonable time the whole DVB-T system, as we will see in the next section.

3 Although the equivalence is not exact, the approximations made are highly accurate, as testified by simulation results shown in Section V.
}

$N_{A} \times 1$ subset of the FFT-transformed thermal noise vector. $\tilde{\mathbf{n}}_{I C I}$ is an "ergodic version" of the vector representing the ICI, with fixed mean power obtained as in (4), while the term $\varphi$ represents a random variable (RV) which takes into account the ICI average power distribution over different OFDM symbols and is defined as

$$
\varphi=\frac{1}{N}\left\|\mathbf{n}_{I C I}\right\|^{2} .
$$

Although uncoded BER performance (for QPSK) are independent from the channel power delay profile [6][8], the latter has a great impact on the coded performance. We have conveniently expressed it by a single random variable for the ICI. Specifically, the RV $\varphi$ accounts for the effects of a frequency selective channel on the ICI. By simulation, we have found that $\varphi$ is roughly distributed as the sum of the (normalized) taps powers, which are exponential RVs. As a consequence, we have simply generated $\varphi$ as the sum of exponential RVs, instead of using (8), which would have been required the complete generation of the vector $\mathbf{n}_{I C I}$. On the other hand, all the time selectivity effects are encompassed in the term $\tilde{\mathbf{n}}_{I C I}$, which is a zero-mean complex Gaussian vector with covariance matrix $\mathbf{C}_{I C I}=E\left[\tilde{\mathbf{n}}_{I C I} \tilde{\mathbf{n}}_{I C I}^{H}\right]=\mathbf{G P F C} \mathbf{C}_{T} \mathbf{F}^{H} \mathbf{P}^{T} \mathbf{G}^{T}$, where $\mathbf{G}$ is a suitable sub-matrix of $\mathbf{I}$ accounting for guard bands removal, $\mathbf{P}$ is a permutation matrix that represents the interleaving, and $\mathbf{C}_{T}$ is the (diagonal) covariance matrix of the ICI in the time domain with entries:

$$
\left[\mathbf{C}_{T}\right]_{k, k}=P_{I C I}-\frac{2}{N} \sum_{m=1}^{N} J_{0}\left(2 \pi f_{D} T_{S}(k-m)\right), \quad 1 \leq k \leq N,
$$

where $J_{0}(\tau)$ is the zero-order Bessel function of the first kind. It is intuitive that the ICI (and then $\varphi$ ) affects the BER performance at high SNR only, whereas for low-to-medium SNR, frequency selectivity impacts on the BER performance through the matrix $\mathbf{H}_{E F D O M}^{(D V B-T)}$. In fact $\mathbf{d}_{E F D O M}=\operatorname{diag}\left(\mathbf{H}_{E F D O M}^{(D V B-T)}\right)$ is a complex Gaussian vector with zero mean and correlation matrix expressed by

$$
\mathbf{C}_{E F D O M}=E\left[\mathbf{d}_{E F D O M} \mathbf{d}_{E F D O M}^{H}\right]=\mathbf{G P F} \boldsymbol{\Sigma} \mathbf{F}^{H} \mathbf{P}^{T} \mathbf{G}^{T},
$$

where $\boldsymbol{\Sigma}$ is a diagonal matrix with $[\Sigma]_{l, l}=\sigma_{l}^{2}, 0 \leq l \leq L-1$, and $[\Sigma]_{l, l}=0, l \geq L$. The parametrization of (10) is important because the BER performance of convolutional codes is strictly related to the matrix $\mathbf{C}_{\text {EFDOM }}$ [20][21]. Thus, in order to simulate the actual OFDM system by our EFDOM, we can generate the useful channel $\mathbf{d}_{E F D O M}$ from an AWGN vector by imposing the colour summarized by $\mathbf{C}_{E F D O M}$ with standard eigenvalue decomposition (EVD) approaches [22]. The simplified EFDOM, through the matrix $\mathbf{H}_{E F D O M}^{(D V B-T)}$, represents the system after the symbol deinterleaver ${ }^{4}$, thus all the other parts are replicated exactly as in the original OFDM system.

It is worth noting that while $\mathbf{C}_{\text {EFDOM }}$ depends only on the power delay profile of the channel, $\mathbf{C}_{I C I}$ depends only on the temporal autocorrelation function of the channel. This means that we have separated the effects of time and frequency selectivity on the ICI, representing them in a meaningful and sufficiently accurate manner, while preserving a simple and easy simulation strategy.

For DAB, differently from DVB-T, we cannot consider each group of OFDM blocks contained in the interleaver

\footnotetext{
${ }^{4}$ For simplicity, in the simplified model bit interleaving is simulated.
} 
length, because the size of the corresponding channel matrix would be unmanageable, given the depth of the interleaving. Nevertheless, the DAB simplified model is expressed by

$$
\mathbf{y}^{(D A B)}=\mathbf{H}_{E F D O M}^{(D A B)} \mathbf{x}_{C}+\mathbf{w}+\sqrt{\varphi} \tilde{\mathbf{n}}_{I C I},
$$

where $\mathbf{x}_{C}$ is the output of the interleaver. In order to derive $\mathbf{H}_{E F D O M}^{(D A B)}$, we make the approximation

$$
\begin{array}{r}
E\left[\left(\frac{1}{N} \sum_{i=L_{C P}+1}^{N+L_{C P}} h_{l}[i]\right)\left(\frac{1}{N} \sum_{i=L_{C P}}^{N+L_{C P}} h_{l}^{*}\left[i+k\left(N+L_{C P}\right)\right]\right)\right]= \\
=E\left[h_{l}[i] h_{l}^{*}\left[i+k\left(N+L_{C P}\right)\right]\right],
\end{array}
$$

This means that we can generate the $l$-th discrete-time channel tap $h_{l}[k]$ by imposing an equivalent maximum Doppler frequency $\tilde{f}_{D}=\left(N+L_{C P}\right) f_{D}$. Thus, we consider a single channel impulse response for each OFDM block, and we generate by DFT-processing the channel frequency response $\mathbf{d}_{E F D O M}^{(D A B)}=\operatorname{diag}\left(\mathbf{H}_{E F D O M}^{(D A B)}\right)$. Clearly, $\mathbf{d}_{E F D O M}^{(D A B)}$ represents a noninterleaved channel, so the simplified simulation model has to explicitly include, differently from the DVB-T one, also the interleaving. However, we have used a simple random interleaver which acts on 400 OFDM blocks (much shorter than the DAB one, which spans 2160 blocks). The random interleaver does not add appreciable complexity to the model and yields the same BER performance, due to the high Doppler spreads considered in this work, as we have verified by simulation. The other terms in (11) are generated similarly to those of DVB-T, but, due to the deep interleaver of DAB, it is possible to neglect the autocorrelation of $\tilde{\mathbf{n}}_{I C I}$, which is generated as an AWGN vector, with power given by (4). Thus the ICI is affected only by frequency selectivity (through the term $\varphi$ ), while the useful channel is affected by both time and frequency selectivity, whereas for DVB-T it was just the other way around. We thus maintain the simplicity and efficiency of the DVB-T simplified system, even if the representation is less meaningful, with still very good accuracy, as shown in the following simulation results.

\section{Simulation Results}

\section{A. EFDOM Accuracy}

Figures 1-2 show the very good agreement of the BER performance in Rayleigh and Rice fading scenarios, respectively, for the actual OFDM systems and the corresponding EFDOM we introduced. For both Figures 1 and 2, DVB-T ( $5 \mathrm{MHz}$ ) and DAB Viterbi performance are plotted. The carrier frequency is $1.4 \mathrm{GHz}$. Code rates $1 / 2$ for DVB-T and $2 / 3$ for DAB are considered, because this choice yields a very similar spectral efficiency (see Table I). The simulation time is reduced by roughly a factor of 2 for both DVB-T and $\mathrm{DAB}$ in RA channels, while the reduction factor is much higher (about 8 for DAB and 15 for DVB-T) in BU channels, because the bottleneck of the OFDM simulation is the generation of the channel matrix in the time-delay domain, which is avoided in the EFDOM. The simulations has been carried out in a MATLAB ${ }^{\circledR} 7$ environment with a Xeon ${ }^{\circledR} 3$ $\mathrm{GHz}$ with $2 \mathrm{~GB}$ of RAM. Except for the channel generation and the convolution with the data, EFDOM and "true" OFDM codes are nearly identical. Consequently, the efficiency gain is obtained by reducing the time needed for generating the channel matrix, which can have up to three millions of elements per iteration in the latter case, while in the former has less than 30000 elements per iteration.

\section{B. Rayleigh Channels with High Delay Spread}

We consider the COST 207 channel BU, which is characterized by Rayleigh fading and high delay spread [9]. Figure 3 plots the Viterbi BER of a DAB and an inner DVB-T system $(8 \mathrm{MHz})$ in the BU channel, for very high speeds, 150 and $300 \mathrm{~km} / \mathrm{h}$, and a carrier frequency $f_{C}=800 \mathrm{MHz}$. This leads to normalized Doppler spreads $\bar{f}_{D} \cong 0.025 \div 0.05$ for DVB-T and $\bar{f}_{D} \cong 0.019 \div 0.038$ for DAB. In the same figure it can be observed that DVB-T achieves better BER performance than DAB (roughly $1 \mathrm{~dB}$ of SNR gain), at low SNR, when used with the same rate $2 / 3$ convolutional code and with LI, although DVB-T exhibits a higher BER floor due to the ICI. The advantage of DVB-T at low-to-medium SNR increases significantly when using LSI. The SNR gap between the two systems become much higher when a similar spectral efficiency is considered, and even the ICI floor is much lower for DVB-T. When using the same rate $1 / 2$ convolutional code, DAB outperforms DVB-T with LI, and, for high SNR, even with LSI. However, the spectral efficiency of DVB with rate $1 / 2$ convolutional code is very similar to that of $\mathrm{DAB}$ with rate $2 / 3$, which has far worse performance. It is worth noting that the BER for DVB-T will be greatly reduced by the outer RS code, making DVB-T BER performance much better than the $\mathrm{DAB}$ one. Figure 3 illustrates also the impact of channel estimation on the DVB-T performance: for both the rates $1 / 2$ and $2 / 3$ of the convolutional code DAB outperforms DVB-T with LI, whereas, when LSI is used, the opposite holds true. Moreover, it can be seen that a perfect channel estimation would grant more than $1 \mathrm{~dB}$ additional gain.

Although DAB has a smaller normalized Doppler spread $\bar{f}_{D}$ than DVB (hence a smaller ICI power) and a far more efficient interleaver, it provides for a channel estimate which is only implicit in the DQPSK demodulation. Anyway, this implicit channel estimation provided by DQPSK is poor for fast time-varying channels, and this fact results to be more important than the advantage in ICI powers and time diversity. In fact, a long power delay profile produces a considerable frequency diversity, and, in the case of DVB-T, this partially compensates for the lower time diversity provided by the use of shorter interleavers than for DAB.

\section{Rice Channels with Low Delay Spread}

Channels with a maximum excess delay comparable with the sampling period can be represented with few taps in the delay domain. For example, the RA channel of COST 207 [9] has $L \cong 4 \div 7$ for DVB-T and $L=2$ for DAB. Thus, the consequent high frequency correlation can largely degrade the DVB-T BER performance, making it worse than for DAB. In fact, this high correlation between the channel coefficients on several contiguous subcarriers of a single OFDM symbol makes frequency interleaving scarcely effective, leading to the presence of error bursts, which degrade the error correcting capability of convolutional codes [19]. This means that the fading experienced by the transmitted signal, in the absence of an effective (long) time interleaver, becomes similar to block fading. This effect can be noticed especially for DVB-T, 
which has no time interleaving at all. For instance, for the RA profile, the correlation coefficient between two subcarriers of the same DVB-T OFDM symbol is always above 0.65 . In DAB systems, this correlation coefficient is even much higher, but the time interleaver, which spans 400 OFDM blocks, greatly reduces the effects of this correlation. Indeed, such a long interleaver makes it possible to consider the channel almost ergodic. This greatly mitigates a deep channel fade, which turns out to be much less significant than for DVB-T, which on the contrary experiences a substantial BER degradation. Due to the previous considerations, partially surprisingly, it turns out that DVB-T performance in (long) Rayleigh channels is better than in (short) Rice channels, even when the Rice factor is higher than $5 \mathrm{~dB}$ (see Figure 3).

Figure 4 displays the BER of DAB and DVB-T $(5 \mathrm{MHz})$ on a Rice (RA) channel with a high Rice factor (i.e., $K=10$ $\mathrm{dB})$, for two high speeds $(v=150$ and $v=300 \mathrm{Km} / \mathrm{h})$ and carrier frequency $f_{C}=1.4 \mathrm{GHz}$. This corresponds to normalized Doppler spreads $\bar{f}_{D} \cong 0.034 \div 0.067$ for DAB and $\bar{f}_{D} \cong 0.07 \div 0.139$ for DVB-T. It can be observed that DAB largely outperforms DVB-T with LI for high SNR, because can exploit a much longer time interleaving. Moreover, the DVB-T performance is better with higher speeds, despite the augmented ICI, due to the higher time diversity offered by the channel. It can be also noticed that, for DVB-T, LSI provides for more than $2 \mathrm{~dB}$ gain.

It is worth noting the behaviour of DVB-T on a Rice RA channel with $K=10$, because it well shows the importance of the time interleaving in this type of channels: indeed, DVB-T achieves better performance for $v=300 \mathrm{Km} / \mathrm{h}$ than for $v=150$, except for the LSI case, despite the fact of the augmented ICI power.

Moreover, even the RS code is scarcely effective in this type of channels, especially for low Doppler spreads. This is because, in channels with high line-of-sight component, the ICI has a reduced impact on the BER performance, while the enhanced Doppler spread makes more effective the DVB-T interleaver in providing for time diversity.

Figure 5 plots the performance of DAB for the RA (Rice with $K=0 \mathrm{~dB}$ ) and for the BU (Rayleigh), for rates $1 / 2$ and $2 / 3$ of the convolutional code, and for two maximum Doppler spreads values that correspond to $v=150$ and $v=300 \mathrm{Km} / \mathrm{h}$ with carrier frequency $f_{C}=1.4 \mathrm{GHz}$. It is worthwhile to note the behaviour of the DAB system in a Rice channel: differently from the DVB-T case, the channel power delay profile has a little effect on the BER performance, thanks to the long interleaver, and, especially for the high Doppler spread, the performance is significantly better in the Rice channel rather than in the Rayleigh one, even when the Rice factor is modest.

\section{CONCLUSIONS}

This paper has presented a novel simulation model, called EFDOM, that permits to efficiently evaluate the BER of OFDM-based broadcasting standards in high-mobility scenarios. By exploiting of this model, the coded BER performances of DAB and DVB-T subject to fast fading have been compared, in both Rayleigh and Rice channels. Simulation results have shown that for DVB-T the frequencyselectivity of the channel has a significant impact on the BER performance, whereas for DAB the main difference is caused by time selectivity and Rice factor. One of the key point of our work is that EFDOM is able to reduce the simulation time of a factor from 2 to 15 (depending on the channel model and on the code rate) with respect to the whole simulation of the true OFDM model. Another key point of our work is that, to our knowledge, this is the first paper that presents a very deep comparison between DAB and DVB-T in mobile scenarios.

In addition, the proposed model open the way to an analytical evaluation of BER performance of coded OFDM systems in high Doppler scenarios, which will be the subject of future work.

\section{REFERENCES}

[1] ETSI, Digital Video Broadcasting (DVB); Framing structure, channel coding and modulation for digital terrestrial television, ETSI EN 300 744 V1.5.1, Nov. 2004.

[2] ETSI, Radio Broadcasting Systems; Digital Audio Broadcasting (DAB) to mobile, portable and fixed receivers, ETSI EN 300401 V1.3.3, May 2001 .

[3] L. J. Cimini, "Analysis and simulation of a digital mobile channel using orthogonal frequency division multiplexing", IEEE Trans. Commun., vol. 33, pp. 665-675, July 1985.

[4] M. Russell and G. L. Stüber, "Interchannel interference analysis of OFDM in a mobile environment", Proc. IEEE Veh. Tech. Conf., vol. 2, July 1995 , pp. 820-824.

[5] P. Robertson and S. Kaiser, "The effects of Doppler spreads in OFDM(A) mobile radio systems", Proc. IEEE Veh. Tech. Conf. Fall, vol. 1, Sept. 1999, pp. 329-333.

[6] E. Chiavaccini and G. M. Vitetta, "Error performance of OFDM signaling over doubly-selective Rayleigh fading channels", IEEE Commun. Lett., vol. 4, pp. 328-330, Nov. 2000.

[7] Y. Li and L. J. Cimini, "Bounds on the interchannel interference of OFDM in time-varying impairments", IEEE Trans. Commun., vol. 49, pp. 401-404, Mar. 2001.

[8] X. Cai and G. B. Giannakis, "Bounding performance and suppressing intercarrier interference in mobile wireless OFDM", IEEE Trans. Commun., vol. 51, pp. 2047-2056, Dec. 2003.

[9] COST 207, Digital Land Mobile Radio Communications, Final Report, Office for Official Publications of the European Communities, Luxembourg, 1989.

[10] P. Bello, "Characterization of randomly time-variant linear channels," IEEE Trans. Commun., vol. 11, pp. 360-393, Dec 1963.

[11] Y. R. Zheng and C. Xiao, "Improved models for the generation of multiple uncorrelated Rayleigh fading waveforms", IEEE Commun. Lett., vol. 6, pp. 256-258, June 2002.

[12] L. Rugini, P. Banelli, and G. Leus, "Simple equalization of time-varying channels for OFDM", IEEE Commun. Lett., vol. 9, pp. 619-621, July 2005.

[13] W. C. Lee, H. M. Park, and J. S. Park, "Viterbi decoding method using channel state information in COFDM system", IEEE Trans. Consum. Electron., vol. 45, pp. 533-537, Aug. 1999.

[14] L. Deneire, P. Vandenameele, L. van der Perre, B. Gyselinckx, and M. Engels, "A low-complexity ML channel estimator for OFDM", IEEE Trans. Commun., vol. 51, pp. 135-140, Feb. 2003.

[15] S. M. Kay, Foundamentals of Statistical Signal Processing: Estimation Theory, Prentice-Hall, Inc., Upper Saddle River, NJ, USA, 1993.

[16] K.-M. Lee, D.-S. Han, and K.-B. Kim, "Performance of the Viterbi decoder for DVB-T in Rayleigh fading channels", IEEE Trans.Consum. Electron., vol. 44, pp. 994-1000, Aug. 1998.

[17] A. G. Armada, B. Bardon, and M. Calvo, "Parameter optimization and simulated performance of a DVB-T digital television broadcasting system", IEEE Trans. Broadcasting., vol. 44, pp. 131-138, Mar. 1998.

[18] Y. Wang, J. H. Ge, B. Ai, P. Liu, and S. Y. Yang, "A soft decision decoding scheme for wireless COFDM with application to DVB-T", IEEE Trans. Consum. Electron., vol. 50, pp. 84-88, Feb. 2004.

[19] J. G. Proakis, Digital Communications , 4th ed., McGraw-Hill, New York, 2001

[20] J. Jootar, J. R. Zeidler, and J. G. Proakis., "Performance of finite-depth interleaved convolutional codes in a Rayleigh fading channel with noisy 
channel estimates", Proc. IEEE Veh. Tech. Conf. Spring, vol. 1, May/June 2005, pp. 600-605.

[21] J. K. Cavers and P. Ho, "Analysis of the error performance of trelliscoded modulations in Rayleigh-fading channels", IEEE Trans. Commun., vol. 40, pp. 74-83, Jan. 1992.

[22] G. H. Golub and C. F. Van Loan, Matrix Computations, 3rd ed., Johns Hopkins Univ. Press, 1996.

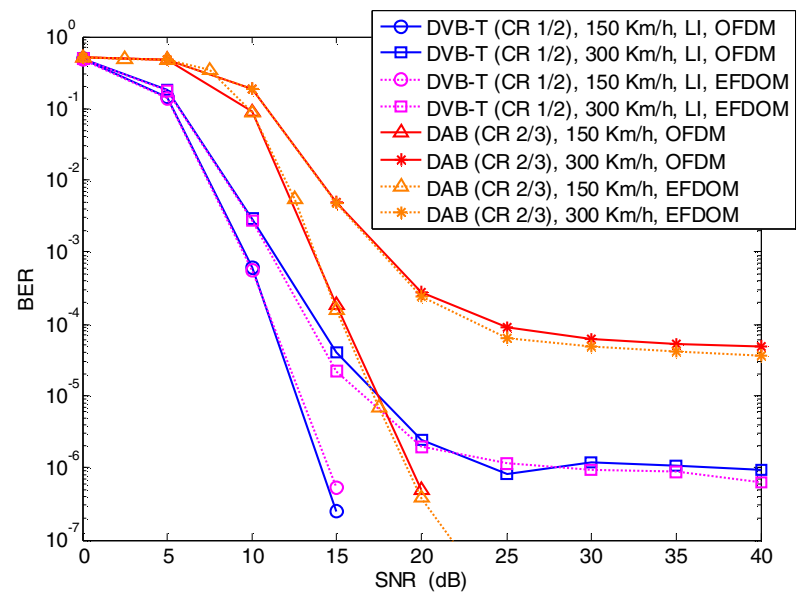

Figure 1. OFDM and EFDOM, Rayleigh (BU) channel, DVB-T $5 \mathrm{MHz}$, Viterbi performance, carrier frequency $1.4 \mathrm{GHz}$.

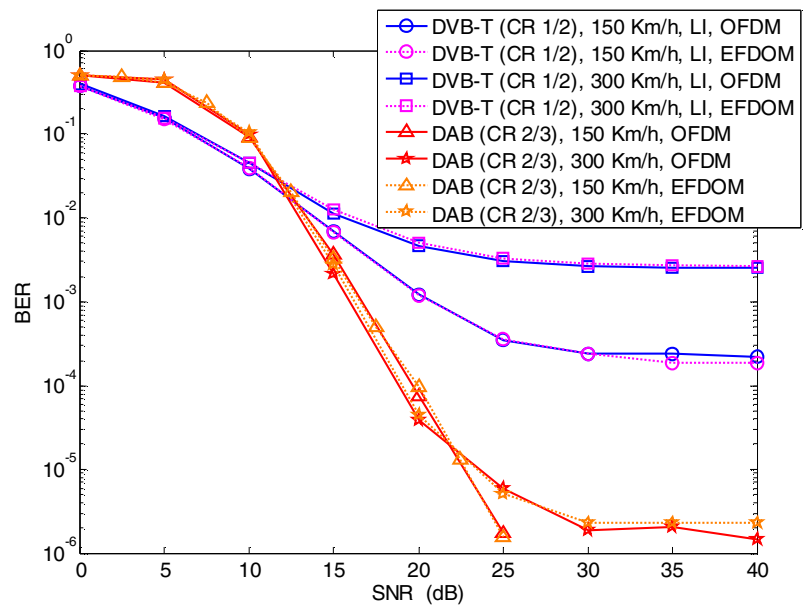

Figure 2. OFDM and EFDOM, Rice (RA) channel with $K=0 \mathrm{~dB}$, DVB-T $5 \mathrm{MHz}$, Viterbi performance, carrier frequency $1.4 \mathrm{GHz}$.

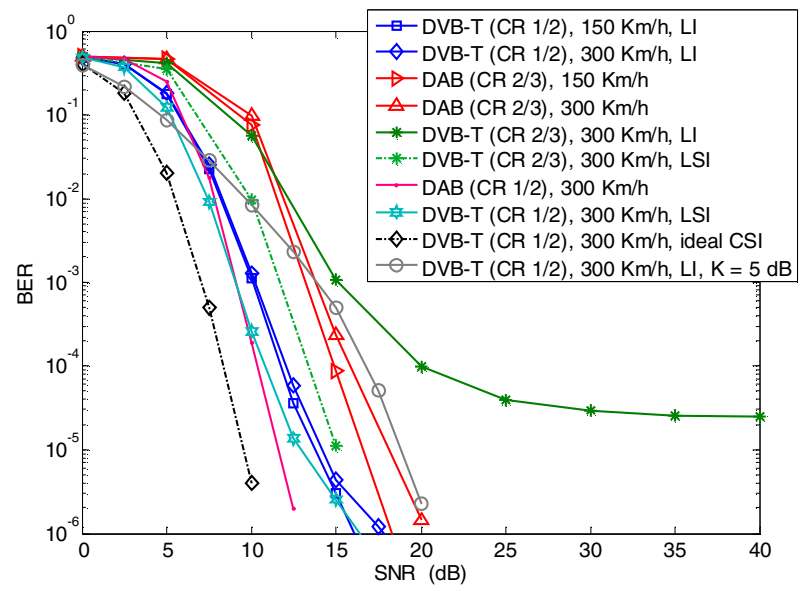

Figure 3. DVB-T $8 \mathrm{MHz}$ (Viterbi) and DAB performance on a Rayleigh (BU) channel (RA for the last curve, where the Rice factor $K$ is specified), carrier frequency $800 \mathrm{MHz}$.

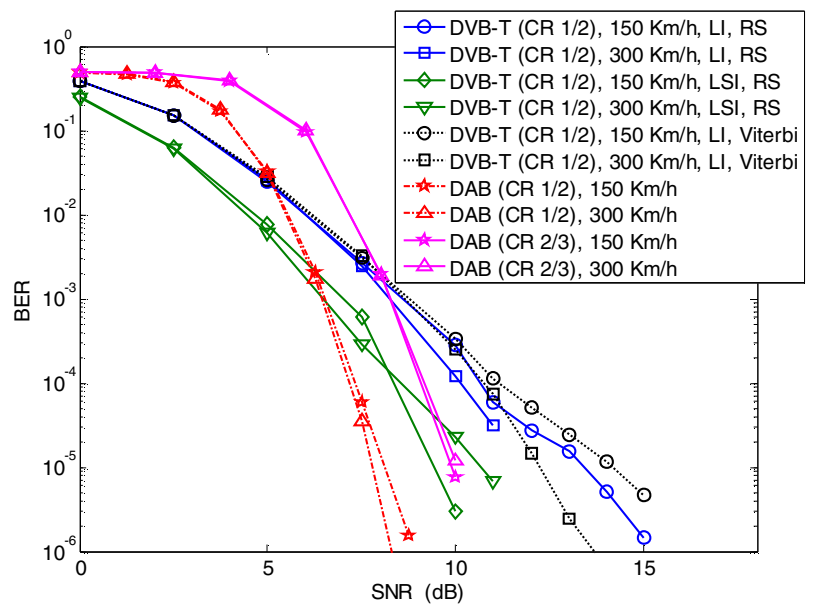

Figure 4. DVB-T and DAB performance on a Rice (RA) channel with $K=10$ $\mathrm{dB}$, carrier frequency $1.4 \mathrm{GHz}$.

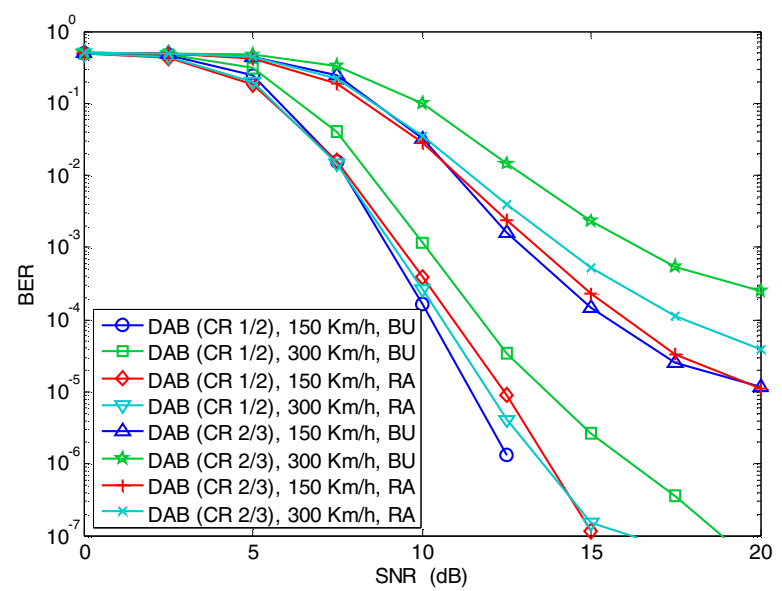

Figure 5. DAB performance, for different Doppler spreads and different rates of the convolutional code, carrier frequency $1.4 \mathrm{GHz}$. 